British Journal of Nutrition (2022), 127, 1441-1442

(c) The Author(s), 2021. Published by Cambridge University Press on behalf of The Nutrition Society. This is an Open Access article, distributed under the terms of the Creative Commons Attribution licence (http://creativecommons.org/licenses/by/4.0/), which permits unrestricted re-use, distribution, and reproduction in any medium, provided the original work is properly cited.

\title{
Editorial
}

\section{Nutrition and COVID-19}

At the end of December 2019, the world became aware of a new respiratory disease following reports of a cluster of cases of 'viral pneumonia' in Wuhan, China. We now know this disease as COVID-19 which is caused by a coronavirus called severe acute respiratory syndrome coronavirus 2 (SARS-CoV-2). This is the 3 rd time in two decades that a highly pathogenic coronavirus has jumped from an animal host to humans - the others were SARS-CoV (in 2003) and MERS-CoV (in 2012). Unlike these earlier coronavirus diseases, COVID-19 has developed into a major global pandemic with more than 211 million cases leading to over 4.4 million deaths so far (21 August 2021).

Although the complex interrelationships between nutrition and the immune system are well established ${ }^{(1)}$, in the early stages of the pandemic little attention was paid to dietary and other lifestyle characteristics of those at risk. However, as the pandemic gathered pace, it became apparent that obesity was a risk factor for COVID-19 - those with obesity were more likely to be diagnosed with COVID-19 and to have more severe disease ${ }^{(2)}$. Since obesity had been linked with more severe infectious respiratory disease as a result of the 2009 pandemic influenza A (H1N1) disease ${ }^{(3)}$, the association with COVID-19 should not have been a surprise.

To support the global effort to address the COVID-19 pandemic, the BJN implemented an expedited review process for relevant manuscripts on nutritional aspects of the disease. Following publication in the BJN, accepted papers have been included in a special COVID-19 collection that was developed by our publishers Cambridge University Press (CUP) - see: https://www.cambridge.org/core/browse-subjects/medicine/ coronavirus-free-access-collection. In addition, we commissioned a number of internationally recognised nutrition experts to write review articles addressing the mechanisms through which specific nutrients or nutritional status may influence susceptibility to SARS-CoV-2 infection and/or disease severity in COVID-19. These 7 papers, along with a selection of non-commissioned articles, are now available in a Special Collection in the BJN, 'Nutrition and COVID-19: Potential mechanisms influencing risk and disease severity'.

In this Special Collection, Gleeson et al. ${ }^{(4)}$ explore the mechanisms through which excess body fat may increase susceptibility to SARS-CoV-2 infection and the development of COVID-19. They propose that obesity and obesity-associated factors such as 'meta-flammation' (a state of chronic inflammation), dietary fat intake and paradoxical suppression of the innate immune response within the pulmonary compartment may be crucial determinants in the host response to a novel viral pathogen ${ }^{(4)}$.
The SARS-CoV-2 virus enters cells via the angiotensinconverting enzyme 2 receptor that is expressed on the plasma membrane of cells in multiple tissues, including the gastrointestinal tract. This raises the possibility of SARS-CoV-2 gastrointestinal infection and the potential for faecal-oral transmission ${ }^{(5)}$. Walton et $a l^{(6)}$ discuss emerging evidence that suggests a link between SARS-CoV-2 infection and the gut microbiome and the potential for using pre-, pro- and synbiotics to modulate the gut microbiome. If successful, this is a potential strategy for supporting the immune function and reducing inflammatory status and so helping in defence against COVID-19(6).

In their review of micronutrient status and COVID-19 risk, Richardson and Lovegrove ${ }^{(7)}$ draw attention to vitamins $\mathrm{A}, \mathrm{C}$ and $\mathrm{D}$, particular $\mathrm{B}$ vitamins (folate, vitamin $\mathrm{B}_{6}$ and vitamin $\mathrm{B}_{12}$ ), and specific trace elements (including $\mathrm{Fe}, \mathrm{Cu}$, Se and $\mathrm{Zn}$ ) that contribute to the normal functions of the immune system. They conclude that avoiding nutrient deficiencies, identifying population groups at high risk of suboptimal nutritional status and the implementation of practical, safe and effective nutrition policy solutions may help strengthen resilience against COVID19. This topic is expanded by Stephenson and Lietz ${ }^{(8)}$ who discuss the role of vitamin A in maintaining both innate and adaptive immunity that is essential in promoting clearance of a primary viral infection and in minimising risks from secondary infections. Then, using the example of measles (caused by the Measles morbillivirus), they review the evidence that vitamin A deficiency may increase the severity of viral disease and that appropriately timed supplementation during recovery may reduce mortality and hasten recovery ${ }^{(8)}$.

Noting that Se status is a key determinant of the host response to viral infections, the potential role of Se in COVID-19 is explored by Bermano et $a l .{ }^{(9)}$. In addition to potentially increasing susceptibility to viral infections, Bermano et al. ${ }^{(9)}$ draw attention to the evidence that passage through Se-deficient animals and humans can amplify the rate of acquisition of mutations in the viral genome (using coxsackievirus $\mathrm{B}_{3}$ and influenza viruses as exemplars) resulting in increased pathogenicity. Over the past year, the SARS-CoV-2 virus has continued to evolve leading to the emergence of 'variants of concern', that increase transmissibility and/ or alter antigenicity ${ }^{(10)}$ and that may have implications for the effectiveness of currently used vaccines.

Having drawn attention to the overlap between the characteristics of those at risk for severe progression of COVID-19 with the characteristics of population groups in which $\mathrm{Zn}$ deficiency is common, Wessels et al. ${ }^{(11)}$ discuss how severe pre-existing $\mathrm{Zn}$ 
deficiency may pre-dispose patients to a severe progression of COVID-19. They suggest that $\mathrm{Zn}$ deficiency may exacerbate the effects of COVID-19 in several ways including (i) by disrupting haematopoiesis and disturbing the balance between innate and adaptive immune cells, (ii) by predisposing to a 'cytokine storm' that can lead to multi-organ failure, (iii) by amplifying thrombus formation and hyper-inflammation that can lead to increased risk of thromboembolism and (iv) by reducing mucosal barrier function in multiple organs and tissues ${ }^{(11)}$.

Although the majority of those infected with the SARS-CoV-2 virus have mild to moderate symptoms, a significant proportion develops respiratory failure due to pneumonia ${ }^{(12)}$. Janssen et $a l^{(13)}$ review the role of vitamin $\mathrm{K}$ in the activation of both pro- and anti-clotting factors in the liver and in the activation of extra-hepatically synthesised protein $S$ which may be important in local thrombosis prevention. In addition, they note that matrix Gla protein is a vitamin K-dependent inhibitor of soft tissue calcification and elastic fibre degradation ${ }^{(13)}$. This is the context for their mechanistic hypothesis in which pneumoniainduced vitamin $\mathrm{K}$ depletion leads to falls in activated MGP and protein $\mathrm{S}$ that aggravate pulmonary damage and coagulopathy, respectively ${ }^{(13)}$.

Despite the success of the COVID-19 vaccination programmes, $<25 \%$ of the world's population has been vaccinated to date (21 August 2021). In addition, the emergence of new variants of SARS-CoV-2 virus with increased transmissibility, even in those who have been fully vaccinated, means that this pandemic is far from over. We can expect to see COVID-19 continuing to exact a heavy toll of morbidity and mortality and that this will continue to affect disproportionally the most vulnerable in our communities. Health inequalities will continue to widen, with inadequate diet and poor nutritional status likely to contribute to those inequalities. The nutrition research community has a responsibility to step up our research on nutrition and COVID-19 so that policy makers, and those who design and deliver clinical and public health services, have the best possible evidence on which to base their decisions.

Although most people who are infected with SARS-CoV-2 make a full recovery within 12 weeks, a significant minority have on-going symptoms that include cutaneous, respiratory, cardiovascular, musculoskeletal, mental health, neurologic and renal involvement ${ }^{(14)}$. This 'Long COVID' syndrome has some similarities with chronic fatigue syndrome, also known as myalgic encephalomyelitis, that can be triggered by a viral infection. The evidence base for nutritional intervention to support those with myalgic encephalomyelitis is very poorly developed. Perhaps the emergence of Long COVID will provide a stimulus for nutrition research that benefits not only those with Long COVID but also those with apparently related conditions such as myalgic encephalomyelitis.
John C. Mathers

Human Nutrition Research Centre, Centre for Healthier
Lives, Population Health Sciences Institute, Newcastle
University, Newcastle upon Tyne, UK
email j.mathers@nutritionsociety.org

doi:10.1017/S0007114521003305

\section{References}

1. Calder PC (2013) Feeding the immune system. Proc Nutr Soc 72, 299-309.

2. Popkin BM, Du S, Green WD, et al. (2020) Individuals with obesity and COVID-19: a global perspective on the epidemiology and biological relationships. Obes Rev 21, e13128.

3. Morgan OW, Bramley A, Fowlkes A, et al. (2010) Morbid obesity as a risk factor for hospitalization and death due to 2009 pandemic Influenza $\mathrm{A}(\mathrm{H} 1 \mathrm{~N} 1)$ disease. PLoS One 5, e9695.

4. Gleeson LE, Roche HM \& Sheedy FJ (2021) Obesity, COVID-19 and innate immunometabolism. Br J Nutr 125, 628-632.

5. Gou M, Tao W, Flavell RA, et al. (2021) Potential intestinal infection and faecal-oral transmission of SARS-CoV-2. Nat Rev Gastroenterol Hepatol 88, 269-283.

6. Walton GE, Gibson GR \& Hunter KA (2021) Mechanisms linking the human gut microbiome to prophylactic and treatment strategies for COVID-19. Br J Nutr 126, 219-227.

7. Richardson DP \& Lovegrove JA (2021) Nutritional status of micronutrients as a possible and modifiable risk factor for COVID-19: a UK perspective. Br J Nutr 125, 678-684.

8. Stephenson CB \& Lietz G (2021) Vitamin A in resistance to and recovery from infection: relevance to SARS-CoV2. Br J Nutr 1-10. doi: 10.1017/S0007114521000246.

9. Bermano G, Meplan C, Mercer DK, et al. (2021) Selenium and viral infection: are there lessons for COVID-19? Br J Nutr $\mathbf{1 2 5}$, 618-627.

10. Harvey WT, Carabelli AM, Jackson B, et al. (2021) SARS-CoV-2 variants, spike mutations and immune escape. Nat Rev Microbiol 19, 409-424.

11. Wessels I, Rolles B, Slusarenko AJ, et al. (2021) Zinc deficiency as a possible risk factor for increased susceptibility, severe progression of Corona Virus Disease 19. Br J Nutr 1-19. doi: 10. 1017/S0007114521000738.

12. Attaway AH, Scheraga RG, Bhimraj A, et al. (2021) Severe covid-19 pneumonia: pathogenesis and clinical management. BMJ 372, n436.

13. Janssen R, Visser MPJ, Dofferhoff ASM, et al. (2021) Vitamin K metabolism as the potential missing link between lung damage and thromboembolism in Coronavirus disease 2019. Br J Nutr 126, 191-198.

14. Akbarialiabad H, Taghrir MH, Abdollahi A, et al. (2021) Long COVID, a comprehensive systematic scoping review. Infection. doi: 10.1007/s15010-021-01666-x. 